\title{
Research Paper: Effect of Age, Gender and Hearing Loss on the Degree of Discomfort Due to Tinnitus
}

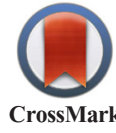

\author{
Maryam Noroozian', Zahra Jafari ${ }^{2,3^{*}}$, Elaheh Shahmirii ${ }^{2,4}$, Shaghayegh Omidvar ${ }^{5}$, Azadeh Zendehbad ${ }^{6}$, Naser Amini ${ }^{7}$, Mahsa Radmehr $^{8}$ \\ Masoud Bagherian', Ali Yoonessi ${ }^{4}$ \\ 1. Department of Memory and Behavioral Neurology, School of Medicine, Tehran University of Medical Sciences, Tehran, Iran. \\ 2. Rehabilitation Research Center, Iran University of Medical Sciences, Tehran, Iran. \\ 3. Department of Basic Sciences, School of Rehabilitation Sciences, Iran University of Medical Sciences, Tehran, Iran. \\ 4. Department of Neuroscience, School of Advanced Technologies in Medicine, Tehran University of Medical Sciences, Tehran, Iran. \\ 5. Department of Audiology, School of Rehabilitation Sciences, Shiraz University of Medical Sciences, Shiraz, Iran. \\ 6. Department of Geriatric Medicine, School of Medicine, Tehran University of Medical Sciences, Tehran, Iran. \\ 7. Cellular and Molecular Research Center, Iran University of Medical Sciences, Tehran, Iran. \\ 8. Department of Audiology, School of Rehabilitation Sciences, Shahid Beheshti University of Medical Sciences, Tehran, Iran \\ 9. Department of Audiology, School of Rehabilitation Sciences, Iran University of Medical Sciences, Tehran, Iran.
}

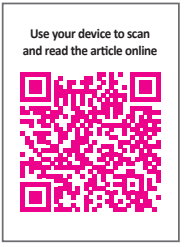

Cftation: Noroozian, M., Jafari, Z., Shahmiri, E., Omidvar, Sh., Zendehbad, A., Amini, N., et al. (2017). Effect of Age, Gender and Hearing Loss on the Degree of Discomfort Due to Tinnitus. Basic and Clinical Neuroscience, 8(6), 435-442. https:// doi.org/10.29252/NIRP.BCN.8.6.435

\section{https://doi.org/10.29252/NIRP.BCN.8.6.435}

Article info:

Received: 25 May 2016

First Revision: 17 June 2016

Accepted: 21 August 2016
Key Words:

Tinnitus, Gender, Age, Hearing loss, Discomfort

\begin{abstract}
A B S T RA C T
Introduction: Tinnitus is one of the complex symptoms of hearing described as a phantom auditory sensation without any external stimulation. Due to the subjective nature of tinnitus, perception and discomfort of tinnitus vary among the patients. The main aim of this study is to investigate the effects of gender, age and the degree of hearing loss on discomfort due to tinnitus.

Methods: Eighteen patients with tinnitus, aged 21-72 years, ( 9 males and 9 females) were recruited. Tinnitus discomfort was investigated by Tinnitus Handicap Inventory (THI) questionnaire. Psychoacoustic assessments of tinnitus and auditory threshold assessments were evaluated using a 2-channel clinical audiometer.

Results: The results showed no significant correlation between THI scores with loudness matching $(\mathrm{P}=0.187)$, mean of auditory threshold $(\mathrm{P}=0.304)$, gender $(\mathrm{P}=0.93)$ and age $(\mathrm{P}=0.200)$. Also, no significant correlation was found between maximal level of hearing loss and pitch matching $(\mathrm{P}=0.208)$.

Conclusion: The study findings suggests that tinnitus is not correlated with age, gender and hearing loss. Overall, tinnitus is a complicated clinical condition which its real impact and degree of discomfort are unclear. More investigation is needed to clarify the factors involving in tinnitus annoyance.
\end{abstract}

\section{* Corresponding Author:}




\section{Introduction}

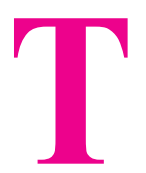

innitus is a phantom auditory feeling that exclusively originates from the nervous system without any corresponding mechanical or vibrating activity in the cochlea (Martines, Bentivegna et al., 2010). The highest prevalence of tinnitus is seen among 40-70 years age range group, and is almost the same for both men and women (Schleuning, 1991). Tinnitus could originate from each segment of the auditory pathway from the cochlea to the auditory cortex (Crummer \& Hassan, 2004). The main obstacle in tinnitus treatment is the lack of any standard method or scale to measure reliably the intensity of tinnitus. The supplementary assessments about tinnitus include pitch matching (adaptation of tinnitus frequency with a pure voice or noise), loudness matching (estimating tinnitus intensity with a pure voice or noise), minimal level of masking (assessing the amount of voice that is needed for masking the tinnitus) and residual inhibition (decreasing or eliminating the tinnitus after exposure to a covering tone of pitch and intensity of tinnitus) (Karatas \& Deniz, 2011).

In addition to the clinical evaluation, questionnaires are usually used for inspecting the individual complaints regarding tinnitus. Various types of questionnaires such as Tinnitus Handicap Questionnaire (THQ), Tinnitus Severity Questionnaire (TSQ) and Tinnitus Reaction Questionnaire (TRQ) have been used (Bahmad, Venosa, \& Oliveii ra, 2006; Hallam, Jakes, Chambers, \& Hinchcliffe, 1985; Kuk, Tyler, Russell, \& Jordan, 1990). Tinnitus Handicap Inventory (THI) is one of the most varied self-assessment tools for tinnitus, which has been considerably used because of its ease of completion, functional and emotional structures, good structural validity, internal cohesion and strong test-retest reliabilities (Karatas \& Deniz, 2011).

The perception of tinnitus differs in various people. It can be perceived as low, high and severe (Valente et al., 2012). Although the tinnitus annoyance and its secondary symptoms are a worldwide healthcare problem and there are numerous requests for therapeutic interventions, the impact of factors such as age, gender and hearing loss on tinnitus remains controversial to date (Seydel, Haupt, Olze, Szczepek, \& Mazurek, 2013).

Gender differences regarding the tinnitus annoyance and its effect on the quality of life have been reported by many researchers (Erlandsson \& Holgers, 2001; Hiller \& Goebel, 2006; Lindgren, Wieslander, Dammström, \& Norbäck, 2009; Meric, Gartner, Collet, \& Chéry-Croze, 1998; Pinto, Sanchez, \& Tomita, 2010; Seydel et al., 2013; Van- neste, Joos, \& De Ridder, 2012; Welch \& Dawes, 2008). Some studies have reported no relationship between gender and nuisance expected from tinnitus (Meric et al., 1998; Pinto et al., 2010; Vanneste et al., 2012), while others have confirmed this kind of relationship (Erlandsson \& Holgers, 2001; Hiller \& Goebel, 2006; Lindgren et al., 2009; Welch \& Dawes, 2008). Moreover, considering that prevalence of hearing loss and tinnitus will increase with senility, several studies have tried to examine the relationship between tinnitus and age (Pinto et al., 2010). However, these studies have failed to reach a unique and specific conclusion. For instance, in one study (Meric et al., 1998), researchers inspected the impact of tinnitus on the quality of life, without finding any association between age and tinnitus annoyance. In another study (Hiller \& Goebel, 2006), more annoyance was reported in older male patients. The impact of hearing loss on the tinnitus annoyance remains a matter of debate.

Several studies have tried to depict the possible association between amount of damage in hearing sensitivity and the scores of the THQ (Axelsson, 1991; Dias \& Cordeiro, 2008; Newman, Jacobson, \& Spitzer, 1996; Jayarajan, Bartlett, \& Ratnayake, 2009; Savastano, 2008; Vallianatou, Christodoulou, Nestoros, \& Helidonis, 2001); however, the results have been contradictory. For instance in Searchfield study, low frequency hearing loss was associated with high level of annoyance caused by tinnitus (using the THQ) (Searchfield, Jerram, Wise, \& Raymond, 2007). In the mentioned study, tinnitus severity index (TSI: A questionnaire that evaluates the tinnitus annoyance) did not correlate with any audiometric findings. This poor communication suggests those patients with tinnitus are heterogeneous and that multiple factors are involved in the impact of these symptoms (Pinto et al., 2010). The relationship between hearing loss and tinnitus pitch has been inspected in several studies and different results have been reported. Some researchers observed a clear relationship between tinnitus pitch and the shape of the audiogram (Jastreboff \& Hazell, 1993; Norena, Micheyl, Chéry Croze, \& Collet, 2002; Roberts, Moffat, \& Bosnyak, 2006; Schecklmann et al., 2012; Sereda et al., 2011), while other studies failed to report such relationship (Pan et al., 2009). Still a matter of debate, several theories about relationship between various types of hearing loss and tinnitus frequency (or pitch) have been proposed (Schecklmann et al., 2012).

Due to the subjective nature of tinnitus, the diversity of causes and the heterogeneity of patients, tinnitus is a complicated issue for study and understanding. Considering these kinds of contradictions, this study aimed to investigate the effects of gender, age and the degree of 
hearing loss on the annoyance caused by tinnitus. Here, the relationship between audiometric data, psychoacoustics assessments related to tinnitus and tinnitus severity were also inspected on the basis of a THI.

\section{Methods}

\subsection{Study participants}

This study was conducted on 18 patients with tinnitus aged from 21 to 72 years [mean(SD) age: 50.22(13.38) years; 9 men]. Participants were selected from patients referred to the Audiology Clinic of Tehran University of Medical Sciences. The study was conducted according to the Helsinki Declaration and its implementation was approved by the Ethics Committee of Tehran University of Medical Sciences. The patients were fully informed about the project and completed consent forms. Inclusion criteria comprised no history of exposure to noise or ototoxic drug use, lack of history of ear disease, neuro-otologic disease, psychiatric disorders, head trauma or accident, brain surgery and consumption of psychotropic drugs (Martines et al., 2010; Martines, Bentivegna, Martines, Sciacca, \& Martinciglio, 2010a; 2010b). To investigate the effect of age, participants were divided into two groups of over and under 50 years old.

\subsection{Audiometry and THI}

Auditory threshold assessments and psychoacoustics evaluations of tinnitus were done using a 2-channel clinical audiometer (Madsen Orbiter 922). Auditory threshold was considered as the average pure tone for frequencies of 500, 1000, 2000, 4000 and $8000 \mathrm{~Hz}$ and was classified as normal hearing $(<20 \mathrm{~dB})$; mild hearing loss (21-40 dB); moderate hearing loss (41-70 dB); severe hearing loss (71-90 dB) and profound hearing loss (>90 dB) (Martines et al., 2010; Martines et al., 2010a; 2010b).

To evaluate the severity of tinnitus and its impact on patient's life, THI (Mahmoudian et al., 2011) was used. THI is a 25 -item questionnaire that contains three subscales, including functional (12 items), emotional (8 items) and catastrophic (5 items) that respectively examine physical functioning, psychological distress, despair and loss of control. Each item can be replied to with three types of answers; 'yes' (4 score), 'sometimes' (2 score) and 'no' (0 score), so that eventually total score ranges from 0 (no disability resulting from tinnitus) to 100 (highest tinnitus annoyance). According to these ratings, we can divide the tinnitus severity into 5 categories: slight (score 0-16), mild (score 18-36), moderate (score 38-56), severe (score 58-76) and catastrophic (score 78100) (Mahmoudian et al., 2011).

\subsection{Psychoacoustic assessments of tinnitus}

For patients who had unilateral tinnitus, the sounds were delivered to the normal side, and for patients with central tinnitus when both ears were affected identically, the right ear was selected to deliver sounds.

\subsection{Loudness matching}

In evaluating tinnitus loudness, the patients were asked to adjust the pure-tone intensity level in $1000-16000 \mathrm{~Hz}$ frequency in such a way that it could be almost equal to the loudness of tinnitus (regardless of the quality of sound). At each frequency, delivering sounds began with an intensity level lower than the patient's hearing threshold at that level of frequency, and then was increased continuously at $1 \mathrm{~dB}$ in steps until the person reported that loudness of external sound corresponded with the tinnitus loudness. To ensure the accuracy of assessment, the loudness matching assessments were repeated at least three times on two different days and the average score of those assessments was recorded as tinnitus loudness on a dBSL scale.

\subsection{Pitch matching}

Pitch matching was conducted using two alternate forced choice questions. Stimuli were presented in pairs and then patients were asked to identify the sound that was most consistent with the frequency of their tinnitus. Before offering each pair of sounds, the intensity level of each was set at a higher level of tinnitus loudness on the basis of the previous adaptive evaluations. Consequently, sounds delivery continued in pairs and in an intermittent manner up until the patient stated that sound frequency was closest to his or her tinnitus pitch.

\subsection{Data analysis}

Statistical analysis was performed considering the ears and not the subjects. Data that belonged to the affected side was inspected in patients with unilateral tinnitus and in patients with bilateral tinnitus both ear scores were analysed (Granjeiro et al., 2008). The KolmogorovSmirnov test was used to assess the normal distribution $(\mathrm{P}<0.05)$. Accordingly, the distributions of THI scores, the frequency with maximum hearing loss and the average auditory threshold were normal, however, it was not normal for loudness matching and pitch matching as- 
sessments. Therefore, an independent samples t-test was administered to compare THI scores between two age groups, as well as, genders. The Spearman correlation coefficient was conducted in order to inspect the correlations between THI scores and loudness matching assessments. It was also applied to determine the correlation between the frequency with the maximum hearing loss and pitch matching assessments. The Pearson correlation was used to determine the correlation between THI scores and the average auditory threshold. $\mathrm{P}$ values are indicated in the tables and related texts for the statistical analyses. All statistical analyses were conducted using SPSS Statistics 22 at a significance level of 0.05 .

\section{Results}

The number of investigated ears was 19 (male=10); six in the right ear, 11 in the left ear, and one was perceived bilaterally. The mean and Standard Deviation (SD) of hearing thresholds was $38.71 \pm 22.78$ in the investigated ears. Two ears were classified as normal hearing sensitivity, 10 with mild, three with moderate and the two with severe hearing loss ( $\mathrm{n}=17$ ears). The audiometric evaluation was not possible in two ears. In addition, the mean and SD of THI scores was $40.53 \pm 24.20$. On the basis of THI scores, the tinnitus severity was categorized as slight in four, mild in three, moderate in seven, severe in two, and catastrophic in the two ears. The mean and SD of psychoacoustic assessments (loudness and pitch matching) according to the age and gender are shown in Table 1. This table shows that the tinnitus frequency is much lower in females than males.

In addition, males perceived the tinnitus sound much louder than their female counterparts. Age did not have any effect on the frequency of tinnitus. Besides, the mean and SD of THI scores as a function of age and gender are indicated in Table 2. No significant correlation was found between THI scores with loudness matching $(\mathrm{r}=-0.317 ; \mathrm{P}=0.187)$ and the mean of auditory threshold $(\mathrm{r}=0.265 ; \mathrm{P}=0.304)$. Moreover, no significant correlation was found between the frequency with maximum hearing loss and pitch matching $(\mathrm{r}=0.412 ; \mathrm{P}=0.208)$.

\section{Discussion}

Here, we found no difference in the annoyance caused by tinnitus between men and women. The results of the study supports the findings of those preceding studies which were unable to show any significant correlation (Erlandsson \& Holgers, 2001; Fioretti, Fusetti, \& Eibenstein, 2013; Meric et al., 1998; Pinto et al., 2010; Vanneste et al., 2012). However, Coelho (based on the visual analogue scale) (Coelho, Sanchez, \& Bento, 2004) and Seydel (using the tinnitus questionnaire: TQ) (Seydel et al., 2013) stated that the scores of tinnitus annoyance are significantly higher in women than in men. Differences in the sample size and assessment tools make it difficult to compare the results of the aforementioned studies.

Table 1. Mean and SD of pitch matching and loudness matching according to gender and age

\begin{tabular}{|c|c|c|c|c|c|c|c|}
\hline & & \multicolumn{3}{|c|}{ Pitch Matching (Hz) } & \multicolumn{3}{|c|}{ Loudness Matching (dBSL) } \\
\hline & & Mean & SD & $\mathbf{N}$ & Mean & SD & $\mathbf{N}$ \\
\hline \multirow{2}{*}{ Gender } & Male & 6000 & 3028.21 & 5 & 10.00 & 9.21 & 10 \\
\hline & Female & 2250 & 2991.06 & 8 & 7.00 & 7.31 & 9 \\
\hline \multirow{2}{*}{ Age } & $50 \geq$ & 3875 & 2657.54 & 4 & 11.56 & 9.37 & 10 \\
\hline & $50<$ & 3611.11 & 3913.34 & 9 & 5.90 & 6.51 & 9 \\
\hline
\end{tabular}

SD: Standard Deviation, N: Number of ears evaluated

NEUR SCIENCE

Table 2. Mean and SD of THI scores according to gender and age (Statistically significant at $\mathrm{P}<0.05)$

\begin{tabular}{cccccc}
\hline & & & THI score & & Statistical Result \\
\cline { 3 - 6 } & & Mean & SD & N & $\mathbf{P}^{*}$ \\
\hline \multirow{2}{*}{ Gender } & Male & 41.00 & 23.84 & 9 & 0.93 \\
& Female & 40.00 & 26.04 & 9 & 0.200 \\
\hline
\end{tabular}

SD: Standard Deviation, N: Number of people evaluated

NEUR:SCIENCE 
Prevalence of tinnitus and hearing loss increases with age. THI scores in patients $>50$ years compared to younger people, were higher but not significantly. Tinnitus is a common auditory phenomenon and more can be heard in a quiet area or by increasing the amount of auditory perception. Thus, it seems plausible that in the elderly, because of unemployment and spending more time at home, the perception of tinnitus annoyance is greater compared to younger subjects (Pinto et al., 2010). Hillerand Goebel (Hiller \& Goebel, 2006) has also reported the increasing annoyance of tinnitus in older adults. This is despite the fact that two previous studies reported no effect of age on the tinnitus annoyance (Meric et al., 1998; Pinto et al., 2010).

In our study, there was also no significant correlation between the degree of hearing loss and their discomfort caused by tinnitus (based on THI score). The impact of hearing loss on the severity of annoyance caused by tinnitus remains unclear. Several studies have tried to show the possible association between THI questionnaire scores and degree of hearing loss (Axelsson, 1991; Baskill \& Coles, 1999; Coles, 1984; Dias \& Cordeiro, 2008; Jayarajan et al., 2009; Savastano, 2008; Vallianatou et al., 2001). A group of studies have not found any association between hearing thresholds and severity of tinnitus annoyance (Baskill \& Coles, 1999; Newman et al., 1996; Vallianatou et al., 2001), others have said that people who have a higher hearing threshold gain higher THI scores (Axelsson, 1991; Coles, 1984; Newman et al., 1996; Jayarajan et al., 2009). In contrast, some researchers also said that people who have a lower hearing threshold obtain higher THI scores (Savastano, 2008). However, the results have been different and sometimes contradictory.

According to psychoacoustic assessment (loudness matching) and score THI, there was no significant correlation between THI and intensity of the tinnitus. The high intensity of the tinnitus that is perceived by patients does not mean that patients take it as a severe problem. Sweetow (Sweetow, 1986) suggests that it could be beneficial for the patients to know that there is no relation between the tinnitus loudness and its annoyance. In addition, it would be helpful for the patients to be informed about the role of other factors on the severity and tinnitus annoyance.

Here, we did not find any relationship between the frequency of the tinnitus and frequency of hearing loss on the basis of the psychoacoustics assessment (pitch matching). The findings of this study confirm some earlier studies (Figueiredo et al., 2010; Flores, Teixeira, Rosito, Seimetz, \& Dall'Igna, 2015; Pan et al., 2009), but are in contrast with the results of other studies that have reported a relation between frequency of the tinnitus and frequency of hearing loss (König, Schaette, Kempter, \& Gross, 2006; Martines et al., 2010; Norena et al., 2002; Schecklmann et al., 2012). By considering the fact that $90 \%$ of patients with tinnitus suffer from hearing loss and the most recent theories represent tinnitus as a central neuroplastic changes that occurs following primary cochlear injuries. The existence of a relationship between frequency of hearing loss (or adjacent frequencies) and frequency of the tinnitus is plausible. On the basis of the hypothesis of homeostatic plasticity, the central auditory system preserves neuronal homeostasis in order to maintain stability in the average of firing and neural-coding efficiency. In many cases, sensory deprivation, probably 'neural noise', has been reinforced due to increased gain, and this increases the neural activity in the range of deprived frequency that is eventually perceived as tinnitus (Schecklmann et al., 2012).

It seems that use of a larger sample size, classification of samples into different age groups and gender differentiated analysis can help to draw conclusions and findings into more detailed discussion. Despite the fact that THI is one of the most popular methods for evaluating the severity of tinnitus (due to easy interpretation and use and for addressing various aspects of the patient's life) (Valente et al., 2012), using other valid tinnitus questionnaires along with THI could provide more interesting findings. Investigation of other factors such as personality, the quality of the noise in tinnitus, and duration of the symptoms may help shed a light on the variables that affect the discomfort in tinnitus.

On the basis of the findings this study, gender, age and hearing loss had no effect on the tinnitus annoyance in THI scores. Overall, the data of this study confirmed that tinnitus discomfort cannot be explained by age, gender, and hearing loss. The study of other factors in tinnitus discomfort is suggested.

\section{Acknowledgements}

We thank the patients who participated in our study. This paper is the result of a research project that was financially supported by Rehabilitation Research Center (RRC), Iran University of Medical Sciences (IUMS).

\section{Conflict of Interest}

The authors report no conflicts of interest exists and they are responsible for the content of this article. Informed consent was obtained from all subjects who participated in this study. 


\section{References}

Axelsson, A. (1991). Causes of tinnitus. Paper presented at the $4^{\text {th }}$ International Tinnitus Seminar, Bordeaux, France, 27-30 August 1991.

Bahmad, F. M., Venosa, A. R., \& Oliveira, C. A. (2006). Benzodiazepines and GABAergics in treating severe disabling tinnitus of predominantly cochlear origin. International Tinnitus Journal, 12(2), 140-4. PMID: 17260879

Baskill, J. L., \& Coles, R. R. A. (1999). Relationship between tinnitus loudness and severity. Paper presented at the $6^{\text {th }}$ International Tinnitus Seminar. Cambridge, United Kingdom, 5-9 September 1999.

Coelho, C. C. D. B., Sanchez, T. G., \& Bento, R. F. (2004). [Characteristics Tinnitus in Patients Given Reference Service (Portuguese)]. rq. Otorrinolaringol, 8(3), 216-224.

Coles, R. R. A. (1984). Epidemiology of tinnitus: (1) Prevalence. The Journal of Laryngology E Otology, 98(S9), 7-15. doi: 10.1017/ s1755146300090041

Crummer, R. W., \& Hassan, G. A. (2004). Diagnostic approach to tinnitus. American Family Physician, 69(1), 120-6.

Dias, A., \& Cordeiro, R. (2008). Association between hearing loss level and degree of discomfort introduced by tinnitus in workers exposed to noise. Brazilian Journal of Otorhinolaryngology, 74(6), 876-83. doi: 10.1016/s1808-8694(15)30148-8

Erlandsson, S. I., \& Holgers, K. M. (2001). The impact of perceived tinnitus severity on health-related quality of life with aspects of gender. Noise and Health, 3(10), 39-51. PMID: 12689455

Figueiredo, R. R., Rates, M. A., de Azevedo, A. A., de Oliveira, P. M., \& de Navarro, P. B. (2010). Correlation analysis of hearing thresholds, validated questionnaires and psychoacoustic measurements in tinnitus patients. Brazilian Journal of Otorhinolaryngology, 76(4), 522-6. PMID: 20835541

Fioretti, A., Fusetti, M., \& Eibenstein, A. (2013). Association between sleep disorders, hyperacusis and tinnitus: Evaluation with tinnitus questionnaires. Noise and Health, 15(63), 91. doi: 10.4103/1463-1741.110287

Flores, L., Rosito, L., Seimetz, B., Dall'Igna, C., \& Teixeira, A (2015). Pitch and loudness from tinnitus in individuals with noise-induced hearing loss. International Archives of Otorhinolaryngology, 20(3), 248-53. doi: 10.1055/s-0035-1562935

Granjeiro, R. C., Kehrle, H. M., Bezerra, R. L., Almeida, V. F., André, L. L. S., \& Oliveira, C. A. (2008). Transient and distortion product evoked oto-acoustic emissions in normal hearing patients with and without tinnitus. Otolaryngology-Head and Neck Surgery, 138(4), 502-6. doi: 10.1016/j.otohns.2007.11.012

Hallam, R. S., Jakes, S. C., Chambers, C., \& Hinchcliffe, R. (1985) A comparison of different methods for assessing the "Intensity" of tinnitus. Acta Oto-Laryngologica, 99(5-6), 501-8. doi $10.3109 / 00016488509182253$

Hiller, W., \& Goebel, G. (2006). Factors influencing tinnitus loudness and annoyance. Archives of Otolaryngology-Head $\mathcal{E}$ Neck Surgery, 132(12), 1323-30. doi: 10.1001/archotol.132.12.1323

Jastreboff, P. J., \& Hazell, J. W. P. (1993). A neurophysiological approach to tinnitus: Clinical implications. British Journal of Audiology, 27(1), 7-17. doi: 10.3109/03005369309077884
Jayarajan, V., Bartlett, J., \& Ratnayake, S. A. B. (2009). Could an underlying hearing loss be a significant factor in the handicap caused by tinnitus. Noise and Health, 11(44), 156. doi: $10.4103 / 1463-1741.53362$

Karatas, E., \& Deniz, M. (2011). The comparison of acoustic and psychic parameters of subjective tinnitus. European Archives of Oto-Rhino-Laryngology, 269(2), 441-7. doi: 10.1007/s00405-0111655-2

König, O., Schaette, R., Kempter, R., \& Gross, M. (2006). Course of hearing loss and occurrence of tinnitus. Hearing Research, 221(1-2), 59-64. doi: 10.1016/j.heares.2006.07.007

Kuk, F. K., Tyler, R. S., Russell, D., \& Jordan, H. (1990). The psychometric properties of a tinnitus handicap questionnaire. Ear and Hearing, 11(6), 434-45. doi: 10.1097/00003446-19901200000005

Lindgren, T., Wieslander, G., Dammström, B. G., \& Norbäck, D. (2009). Tinnitus among airline pilots: Prevalence and effects of age, flight experience, and other noise. Aviation, Space, and Environmental Medicine, 80(2), 112-6. doi: 10.3357/ asem.2364.2009

Mahmoudian, S., Shahmiri, E., Rouzbahani, M., Jafari, Z., Reza Keyhani, M., Rahimi, F., et al. (2011). Persian language version of the" Tinnitus Handicap Inventory": Translation, standardization, validity and reliability. International Tinnitus Journal, 16(2), 93-103. PMID: 22249867

Martines, F., Bentivegna, D., Di Piazza, F., Martines, E., Sciacca V., \& Martinciglio, G. (2010). Investigation of tinnitus patients in Italy: Clinical and audiological characteristics. International Journal of Otolaryngology, 2010, 1-8. doi: 10.1155/2010/265861

Martines, F., Bentivegna, D., Martines, E., Sciacca, V., \& Martinciglio, G. (2010a). Assessing audiological, pathophysiological and psychological variables in tinnitus patients with or without hearing loss. European Archives of Oto-Rhino-Laryngology, 267(11), 1685-93. doi: 10.1007/s00405-010-1302-3

Martines, F., Bentivegna, D., Martines, E., Sciacca, V., \& Martinciglio, G. (2010b). Characteristics of tinnitus with or without hearing loss: Clinical observations in Sicilian tinnitus patients. Auris Nasus Larynx, 37(6), 685-93. doi: 10.1016/j. anl.2010.03.008

Meric, C., Gartner, M., Collet, L., \& Chéry-Croze, S. (1998). Psychopathological profile of tinnitus sufferers: Evidence concerning the relationship between tinnitus features and impact on life. Audiology and Neurotology, 3(4), 240-52. doi: $10.1159 / 000013796$

Newman, C. W., Jacobson, G. P., \& Spitzer, J. B. (1996). Development of the tinnitus handicap inventory. Archives of Otolaryngology - Head and Neck Surgery, 122(2), 143-8. doi: 10.1001/ archotol.1996.01890140029007

Norena, A., Micheyl, C., Chéry Croze, S., \& Collet, L. (2002). Psychoacoustic characterization of the tinnitus spectrum: Implications for the underlying mechanisms of tinnitus. Audiology and Neurotology, 7(6), 358-69. doi: 10.1159/000066156

Pan, T., Tyler, R. S., Ji, H., Coelho, C., Gehringer, A. K., \& Gogel, S. A. (2009). The relationship between tinnitus pitch and the audiogram. International Journal of Audiology, 48(5), 277-94. doi: $10.1080 / 14992020802581974$ 
Pinto, P. C. L., Sanchez, T. G., \& Tomita, S. (2010). The impact of gender, age and hearing loss on tinnitus severity. Brazilian Journal of Otorhinolaryngology, 76(1), 18-24. PMID: 20339684

Roberts, L. E., Moffat, G., \& Bosnyak, D. J. (2006). Residual inhibition functions in relation to tinnitus spectra and auditory threshold shift. Acta Oto-Laryngologica, 126(sup556), 27-33. doi: $10.1080 / 03655230600895358$

Savastano, M. (2008). Tinnitus with or without hearing loss: Are its characteristics different. European Archives of Oto-Rhino-Laryngology, 265(11), 1295-1300. doi: 10.1007/s00405-008-0630-z

Schecklmann, M., Vielsmeier, V., Steffens, T., Landgrebe, M., Langguth, B., \& Kleinjung, T. (2012). Relationship between audiometric slope and tinnitus pitch in tinnitus patients: Insights into the mechanisms of tinnitus generation. PLOS ONE, 7(4), e34878. doi: 10.1371/journal.pone.0034878

Schleuning II, A. J. (1991). Management of the patient with tinnitus. Medical Clinics of North America, 75(6), 1225-37. doi: 10.1016/s0025-7125(16)30383-2

Searchfield, G., Jerram, C., Wise, K., \& Raymond, S. (2007). The impact of hearing loss on tinnitus severity. Australian and New Zealand Journal of Audiology, 29(2), 67-76. doi: 10.1375/ audi.29.2.67

Sereda, M., Hall, D. A., Bosnyak, D. J., Edmondson Jones, M., Roberts, L. E., Adjamian, P., et al. (2011). Re-examining the relationship between audiometric profile and tinnitus pitch. International Journal of Audiology, 50(5), 303-12. doi: 10.3109/14992027.2010.551221

Seydel, C., Haupt, H., Olze, H., Szczepek, A. J., \& Mazurek, B. (2013). Gender and chronic tinnitus. Ear and Hearing, 34(5), 661-72. doi: 10.1097/aud.0b013e31828149f2

Sweetow, R. W. (1986). Cognitive aspects of tinnitus patient management. Ear and Hearing, 7(6), 390-6. doi: 10.1097/00003446198612000-00008

Valente, J. P., Pinheiro, L. A., Carvalho, G. M., Guimarães, A. C., Mezzalira, R., Stoler, G., et al. (2012). Evaluation of factors related to the tinnitus disturbance. The International Tinnitus Journal, 17(1), 21-5.

Vallianatou, N. G., Christodoulou, P., Nestoros, J. N., \& Helidonis, E. (2001). Audiologic and psychological profile of Greek patients with tinnitus - Preliminary findings. American Journal of Otolaryngology, 22(1), 33-7. doi: 10.1053/ajot.2001.20689

Vanneste, S., Joos, K., \& De Ridder, D. (2012). Prefrontal cortex based sex differences in tinnitus perception: Same tinnitus intensity, same tinnitus distress, different mood. PLOS ONE, 7(2), e31182. doi: 10.1371/journal.pone.0031182

Welch, D., \& Dawes, P. J. D. (2008). Personality and perception of tinnitus. Ear and Hearing, 29(5), 684-92. doi: 10.1097/ aud.0b013e318177d9ac 
